\title{
How to Keep From Falling Forward: Elementary Swing Leg Action for Passive Dynamic Walkers
}

\author{
Martijn Wisse, Member, IEEE, Arend L. Schwab, Richard Q. van der Linde, and Frans C. T. van der Helm
}

\begin{abstract}
Stability control for walking bipeds has been considered a complex task. Even two-dimensional fore-aft stability in dynamic walking appears to be difficult to achieve. In this paper we prove the contrary, starting from the basic belief that in nature stability control must be the sum of a number of very simple rules. We study the global stability of the simplest walking model by determining the basin of attraction of the Poincaré map of this model. This shows that the walker, although stable, can only handle very small disturbances. It mostly falls, either forward or backward. We show that it is impossible for any form of swing leg control to solve backward falling. For the problem of forward falling, we devise a simple but very effective rule for swing leg action: "You will never fall forward if you put your swing leg fast enough in front of your stance leg. In order to prevent falling backward the next step, the swing leg shouldn't be too far in front." The effectiveness of this rule is demonstrated with our prototype "Mike."
\end{abstract}

Index Terms-Legged locomotion, passive dynamic walking, reflex, swing leg control.

\section{INTRODUCTION}

W HEN designing a practical locomotion system, the engineer usually chooses wheels or tracks. On one hand, legged locomotion seems complicated, even more so with only two legs, when static stability is out of the question. On the other hand, for some reason human beings have been equipped with two legs, and seem to have no difficulty with locomotion. Moreover, walking does not seem to require any attention, as one can concentrate on complicated thoughts while walking. Is bipedal walking then really as complicated as the engineer suspects?

No, it can quite simply be the natural mode of a purely mechanical system. Connect two rods by a hinge, and the system can walk down a shallow slope, the legs swinging in their natural frequency. Patents over 100 years old (e.g., [8]) already use this principle. In 1989, McGeer [17] performed rigorous numerical and practical experiments, showing that passive dynamic

Manuscript received September 9, 2003; revised January 6, 2004. This paper was recommended for publication by Associte Editor H. Arai and Editor A. De Luca upon evaluation of the reviewers' comments. This work was supported by the Dutch Technology Foundation STW.

M. Wisse and A. L. Schwab are with the Department of Mechanical Engineering, Delft University of Technology, 2628CD Delft, The Netherlands (e-mail: m.wisse@wbmt.tudelft.nl).

R. Q. van der Linde is with the Department of Mechanical Engineering, Delft University of Technology, 2628CD Delft, The Netherlands and also with ALTRAN, Hoofddorp NL 3123, The Netherlands.

F. C. T. van der Helm is with the Department of Mechanical Engineering, Delft University of Technology, 2628CD Delft, The Netherlands and also with the Department of Engineering Technology, Twente University, 7500AE Enschede, The Netherlands.

Digital Object Identifier 10.1109/TRO.2004.838030

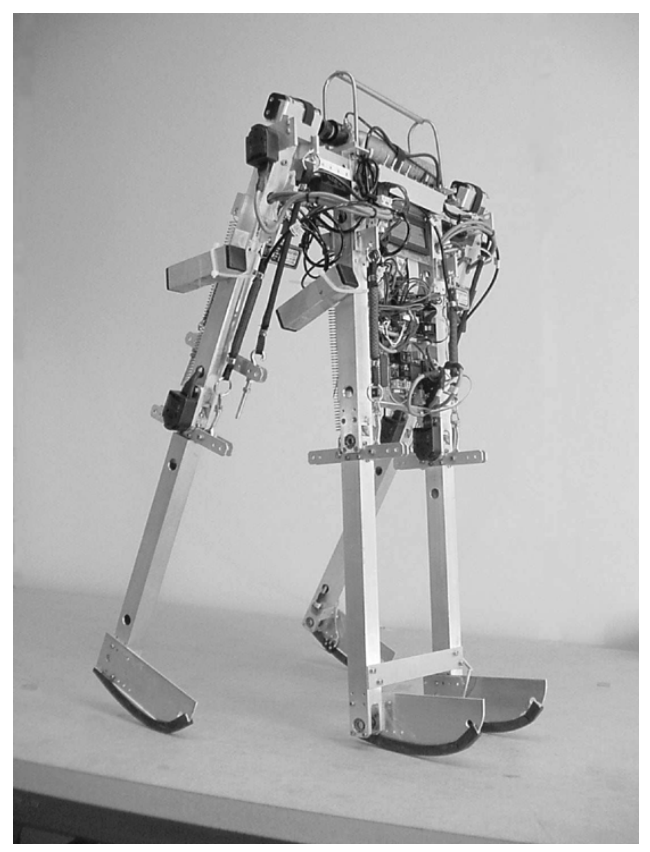

Fig. 1. Prototype Mike: 2-D passive dynamic walking robot with pneumatic McKibben muscles at the hip.

walking, as he termed it, even allows for knees. The key to passive dynamic walking is the repetitive nature of the walking motion, a limit cycle. If such a limit cycle is existent and stable, the walking motion is successful. McGeer, Garcia [10], Van der Linde [23], and Goswami [11] among others researched the influence of different parameters on the stability of such walking cycles. It is now known that by applying round feet, a large hip mass compared to the leg mass, and not too steep a slope, a passive dynamic walker can be constructed that is stable enough for manual startup by an experienced person.

However, human beings can deal with much larger disturbances. On top of the passive locomotory system, humans are actively reacting to perturbations of the walking cycle. We presume that the human control scheme is of the same elegant simplicity as the passive dynamic walking motion. A basic assumption in our research is that the human walking motion is stabilized by a number of very simple, modular control rules. In this paper, we focus on one of those modules; swing leg control. We take the simplest walking model and ask the question: "can we achieve global stability for the simplest walking model with a simple swing leg control rule?". The answer is then validated with our prototype Mike, see Fig. 1. 

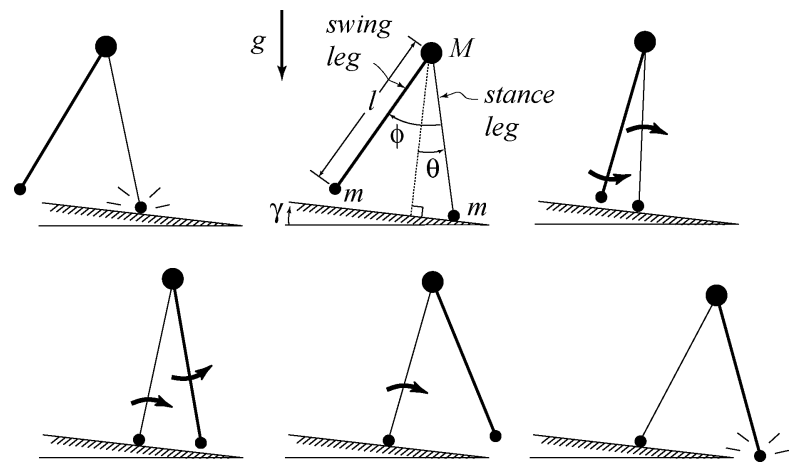

Fig. 2. Typical passive walking step. The new stance leg (lighter line) has just made contact with the ramp in the upper left picture. The swing leg (heavier line) swings until the next heelstrike (bottom right picture). The top-center picture gives a description of the variables and parameters that we use. $\theta$ is the angle of the stance leg with respect to the slope normal. $\phi$ is the angle between the stance leg and the swing leg. $M$ is the hip mass, and $m$ is the foot mass. $l$ is the leg length. $\gamma$ is the ramp slope, and $g$ is the acceleration due to gravity. Reprinted with permission from Garcia et al. [10].

\section{Modeling AND ANALYSIS}

\section{A. Simplest Walking Model}

This research starts with the simplest mechanical model still possessing the ability to perform a bipedal walking motion, as conceived by Garcia et al. [10]. The model, shown in Fig. 2, consists of two rigid links with unit length, connected by a frictionless hinge at the hip. The mass is distributed over three point masses; one with unit mass $M$ at the hip, and two with infinitesimally small mass $m$ at the feet. This unactuated two-link system walks down a slope in a gravity force field with unit magnitude. The scaled model of the walker now only has one free parameter, the slope angle $\gamma$.

A walking step is started with both feet on the slope. The front foot has just made ground contact, the hind foot has a velocity away from the floor. During a step, the stance foot is modeled as a hinge, connected to the floor. The swing foot is moving freely as the other end of a double pendulum. At about midstance, the swing foot is briefly allowed to be below floor level ("foot-scuffing"), which is inevitable for a walker with straight legs. Knees ([1], [6], [18], [26]) or other leg shortening measures ([22]), as well as three-dimensional (3-D) motion ([5], [14], [24]) would solve the problem but increase complexity of the model. After this short through-pass, the second time that the swing foot reaches floor level is regarded as heel-strike, the end of the step. The swing foot makes a fully inelastic collision and becomes the new stance leg. Instantaneously, the former stance leg looses ground contact, and a new step begins.

\section{B. Limit Cycle Analysis}

According to common practice, the dynamic behavior of the simplest walking model is investigated with the following computer simulation procedure. The simulation of one step comprises a smooth leg swing motion, an abrupt collision at heel strike, and the switching of leg function. A simple and efficient method for deriving the necessary equations of motion is given in [20]. Here we will only supply a brief overview of the simulation procedure for one walking step. Then, this procedure is applied to investigate how initial conditions change from one step to the next.

The model has two independent degrees of freedom, the absolute stance leg angle $\theta$, and the relative swing leg angle $\phi$. The equations of motion, as presented in [10] and [20], read

$$
\left[\begin{array}{l}
\ddot{\theta} \\
\ddot{\phi}
\end{array}\right]=\left[\begin{array}{l}
\sin (\theta-\gamma) \\
\sin (\phi)\left(\dot{\theta}^{2}-\cos (\theta-\gamma)\right)+\sin (\theta-\gamma)
\end{array}\right] .
$$

The state of the system at the start of step $n\left(\phi_{n}=2 \theta_{n}\right)$ is completely determined by $\theta_{n}, \dot{\theta}_{n}$, and $\dot{\phi}_{n}$. Shortly it will be clear that also $\dot{\phi}_{n}$ is a dependent initial condition, leaving only two independent initial conditions describing the start of a step. With these initial conditions, the equations of motion are numerically integrated until the end of step $n$ (thus the start of step $n+1$ ) is detected (when again $\phi=2 \theta$ ).

At heel strike just before the start of step $n+1$, the collision of the former swing foot with the floor, simultaneous with the loss of ground contact of the former stance leg, leads to an instantaneous velocity change from the pre-collision state $(-)$ to the post-collision state $(+)$ calculated with

$$
\left[\begin{array}{c}
\dot{\theta}_{n+1}^{+} \\
\dot{\phi}_{n+1}^{+}
\end{array}\right]=\left[\begin{array}{c}
\cos \left(2 \theta_{n+1}\right) \\
\cos \left(2 \theta_{n+1}\right)\left(1-\cos \left(2 \theta_{n+1}\right)\right)
\end{array}\right] \dot{\theta}_{n+1}^{-} .
$$

From the collision equation (2), it is obvious that the initial conditions of the next step are only a function of $\theta$ and $\dot{\theta}$. Therefore, in continuous walking there are only two independent initial conditions. This is a result of the peculiar mass distribution into three point masses. The final part of the simulation is switching the stance and swing leg, resulting in initial conditions for the next step. The simulation has now completed one walking step, and can be repeated with the new initial conditions.

The above simulation procedure is regarded as a step-to-step function $\mathbf{S}$

$$
\left[\begin{array}{c}
\theta_{n+1} \\
\dot{\theta}_{n+1}
\end{array}\right]=\mathbf{S}\left(\left[\begin{array}{c}
\theta_{n} \\
\dot{\theta}_{n}
\end{array}\right]\right)
$$

Monitoring the state of the system only once per cycle like this is known as Poincaré mapping, with the event of heel strike taken as the Poincaré section. The walker is in a limit cycle if, logically, $\left[\theta_{n+1}, \dot{\theta}_{n+1}\right]=\left[\theta_{n}, \dot{\theta}_{n}\right]$. These initial conditions are then a fixed point on the Poincaré map. Fixed points can be found with a Newton-Raphson iteration procedure as described in [10], [17], [20], or [27]. For the simplest walker, we usually find zero or two fixed points, as elaborated in [10]. These fixed points represent an equilibrium of the gravitational energy input of the slope and the collisional energy loss at heel strike. The fixed points are the basis for stability research; if away from a fixed point, will the walker return there over a number of steps?

\section{Linearized Stability, Local Stability}

Suppose we know the fixed point of the simplest walking model, and start the simulation with these initial conditions. By definition, every subsequent step will be equal. Starting away from the fixed point with small errors $\epsilon_{n}$ on the initial conditions results in errors on the initial conditions of the next step 


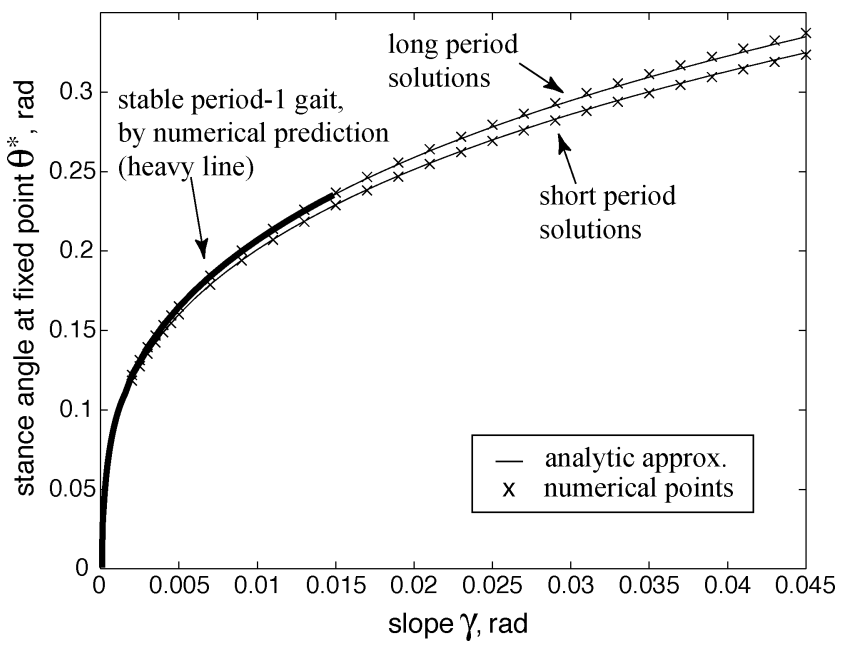

Fig. 3. Stance leg angle $\theta$ at fixed point versus slope angle $\gamma$. In this paper, all simulations are performed with a slope of $\gamma=0.004$ rad leading to a fixed point with stance leg angle $\theta=0.15 \mathrm{rad}$. Reprinted with permission from Garcia $e t$ al. [10].

as $\boldsymbol{\epsilon}_{n+1}$. For small errors, we assume linearity around the fixed point, such that

$$
\boldsymbol{\epsilon}_{n+1}=\mathbf{J} \boldsymbol{\epsilon}_{n} \quad \text { with } \quad \mathbf{J}=\frac{\partial \mathbf{S}}{\partial\left(\theta_{n}, \dot{\theta}_{n}\right)} .
$$

$\mathbf{J}$ is the Jacobian of the stride function $\mathbf{S}$ and is approximately determined by performing the simulation procedure once for both errors $\epsilon_{n}$, one for each independent initial condition. The stability characteristics are described by the two eigenvalues $\lambda_{1}$ and $\lambda_{2}$ of the Jacobian $\mathbf{J}$; if both are smaller than 1 in magnitude, errors decay over subsequent steps. The smaller the eigenvalues, the faster the walker converges toward the fixed point.

Garcia et al. [10] performed this linearized stability analysis on the simplest walker, showing that it has one stable fixed point for small slopes up to $\gamma=0.015 \mathrm{rad}$, and none for steeper slopes, see Fig. 3. This concludes the recapitalization of results from literature. It is important to note that these results are only valid for small errors, therefore only describing the local stability around the fixed point.

\section{Basin of Attraction, Global Stability}

For practical use, one wants to know when the walker keeps walking, and when it falls down. Clearly, the more initial conditions of $[\theta, \dot{\theta}]$ result in continuous walking, the more tolerant is the walker for incorrect launches and in-motion disturbances. The entire collection of initial conditions leading to walking is what is called the basin of attraction. We know that there must be some basin of attraction when the walker is linearly stable around the fixed point, but how large is it? Below, we will describe how to find the complete basin of attraction with the cell mapping method [12], and apply this method to the simplest walking model.

The region of feasible initial conditions is subdivided into a large number $(N)$ of small cells. All unfeasible initial conditions (e.g., $\dot{\theta}>0$ ) are regarded as a small number $(z)$ of very large cells, so called sink cells. The cells are numbered 1 to $N+$ $z$. By application of the step-to-step function $\mathbf{S}$ to the center

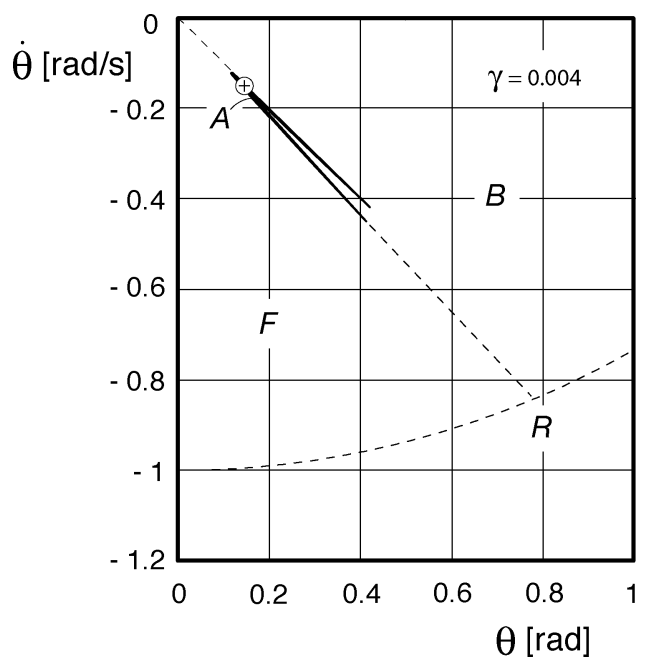

Fig. 4. Poincaré section for the simplest walker with initial stance leg angle $\theta$ and velocity $\dot{\theta}$ together with failure modes; falling Forward, falling Backward, and Running, and the basin of Attraction of the cyclic walking motion $(\theta, \dot{\theta}) \equiv(0.1534,-0.1561)$ (indicated with “+”) at a slope of $\gamma=0.004$ rad. Reprinted from [20].

of each cell, all of the $N+z$ cells point to initial conditions inside one of the other cells, except the sink cells which point to themselves by definition. Starting with cell 1 , a sequence of cells appears by following the pointers. This sequence either ends in a sink cell or in a repetitive cycle. This cycle can consist of one self-repeating cell (a fixed point), or a number of cells (multipleperiod walking, Garcia [9]). The fixed point is identified and all cells in the sequence are labeled as basin of attraction of that fixed point. Then the procedure is repeated with cell 2, then cell 3 , etc. As soon as a known cell (from a previous sequence) is encountered, the current sequence merges with that of the known cell. The procedure is repeated until all cells are labeled.

Application of the cell mapping method results in a list with all attractors (fixed points) and classification of all discretization points into this list. Not only period-one walking gaits can be found, also period- $k$ walking gaits. Results of the cell mapping method are as accurate as the discretization, within these tolerances fixed points may come and go. For example, what appears to be a fixed cell might in fact be slowly changing initial conditions (smaller changes than the discretization) of subsequent steps.

With the cell mapping method, the basin of attraction of the simplest walker is calculated in [20], see Fig. 4. Although the shape and size of the basin of attraction slightly varies for different slope angles, for this article we have chosen to use a representative slope angle of $\gamma=0.004 \mathrm{rad}$. In Fig. 4 the basin of attraction is represented by the very thin area A, otherwise the walker falls forward or backward, or, if started with high speeds, the stance foot loses compressive ground contact (running). This analysis describes the global stability of the walker. This is the more important stability measure for the robustness of the gait [24].

Note that the calculation of the basin of attraction is a costly business as the number of calculations increases with the power of the number of degrees of freedom. This is one of the reasons that we perform the simulation analysis on the simplest walking model instead of on the more complete model of Mike. 


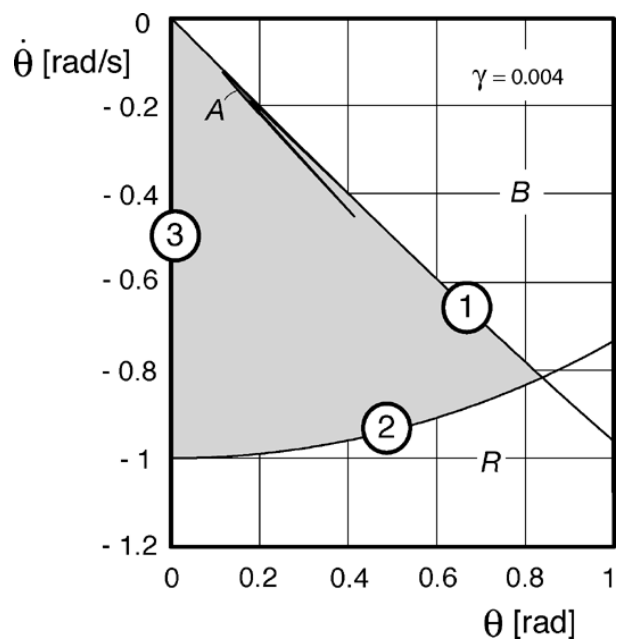

Fig. 5. Maximally obtainable basin of attraction (gray area, bounded by Lines 1,2 and 3, see text) and uncontrolled basin of attraction (thin area A) of the simplest walking model. The entire problem of falling forward can be solved with swing leg control, while the problem of falling backward (area B) remains existent and would need something else than swing leg control.

\section{SWING LEG ACTION FOR A LARGER BASIN OF ATTRACTION}

\section{A. Largest Possible Basin of Attraction}

Now that the basin of attraction of the simplest walker is known, some questions arise. Is it a sufficiently large basin of attraction, or is control necessary? Can control of only the swing leg have any substantial positive effect on the basin of attraction? Before answering these questions, we should recognize that any conceivable hip action does not influence the current stance leg motion whatsoever with the assumption of massless legs. The only thing that matters is the hip angle at heel strike. In other words, swing leg control of the simplest walker can only influence the subsequent step.

Therefore, swing leg control cannot do anything for the walker if the current hip velocity is not enough to pass the apex of the hip trajectory. The mathematical equivalent of this requirement is the following energy inequality:

$$
\frac{1}{2} M(\dot{\theta} l)^{2}>M g l(1-\cos \theta)
$$

or, rewriting and scaling $M, g$, and $l$ to unity

$$
|\dot{\theta}|>2 \sin \frac{\theta}{2} .
$$

This inequality is represented in Fig. 5 with Line (1). Note that this is the familiar separatrix in the normal simple pendulum phase portrait. Also note that Line (1) does not coincide with the dashed boundary between area B and area F in Fig. 4. The area between the two lines represents a set of initial conditions that, for the fully passive walker, does not lead to immediate falling backward, but to a short series of successful steps that eventually leads to falling backward [20].

The second boundary to the initial conditions for making a successful step is the requirement for compressive foot contact: at high velocities the centrifugal effect overcomes gravity and the stance foot would loose contact. This boundary is represented in Fig. 5 as Line (2). The scaled vertical contact force $f_{v}$ is given in [20] as

$$
f_{v}=-\cos (\theta)\left(\dot{\theta}^{2}-\cos (\theta-\gamma)\right)
$$

Requiring that $f_{v}>0$, we get the inequality for Line (2)

$$
|\dot{\theta}|<\sqrt{\cos \theta}
$$

Note that the fact that Line (2) passes through $\{\theta, \dot{\theta}\}=\{0,-1\}$ corresponds to saying that for this nondimensionalized walker the Froude number $\sqrt{v^{2} /(g l)}$ is equal to 1 (with body speed $v$ equal to stance leg velocity $\dot{\theta}$ due to the unit leg length).

The third and last boundary of the maximally achievable basin of attraction is somewhat arbitrary; we only investigate initial conditions with a positive stance leg angle, i.e., starting with the stance foot in front of the swing foot, resulting in Line (3).

Lines (1), (2), and (3) are the outer boundaries for any basin of attraction that swing leg control could possibly achieve for the simplest walker. Therefore we choose to use the area inside these boundaries as a reference for the size of the basin of attraction. Comparing the area of the thin region $A$ in Fig. 4 with the large gray area in Fig. 5, we find that without control, the basin of attraction is only $0.3 \%$ of the maximally achievable. This result justifies the search for a controller to enlarge the basin of attraction.

Summarized so far, we have said that the uncontrolled simplest walker very rarely walks. It mostly falls, either forward or backward. When adding swing leg control, we cannot address the problem of falling backward whatsoever. Therefore, for the swing leg controller we only have to consider the forward falling problem. This makes things easy; as long as we make sure that the swing leg swings forward fast enough, and then just keep it there, the problem should be solved. There is only one extra requirement; the swing leg should not be too far forward, otherwise the walker will fall backward at the subsequent step.

\section{B. Rimless Wheel}

If for now we stick to the idealized situation of a massless swing leg, we could imagine the swing leg controller putting the swing leg at a preset, constant, forward angle immediately after the step has started. The behavior of the walker would then be exactly equal to the "rimless spoked wheel," a system that has a number of "legs" (the spokes) at equal, fixed angles apart (see the inset in Fig. 6). This system has been studied in depth by Coleman [4], who concluded that it would always reach a stable cyclic walking motion, provided that the leg angle is small enough for the floor slope angle. For the slope angle of $0.004 \mathrm{rad}$ that we use throughout this paper, the interspoke angle should be smaller than $0.4 \mathrm{rad}$ [3], otherwise it would slow down and eventually come to a stop and fall backward. We choose a safe value of $0.3 \mathrm{rad}$, i.e., a stance leg angle $\theta=0.15 \mathrm{rad}$ at the start of a step, which corresponds to the natural gait of the simplest walking model at this slope (see Fig. 3). Fig. 6 shows the basin of attraction of the rimless wheel with this interspoke angle of $2 \cdot 0.15 \mathrm{rad}$. 


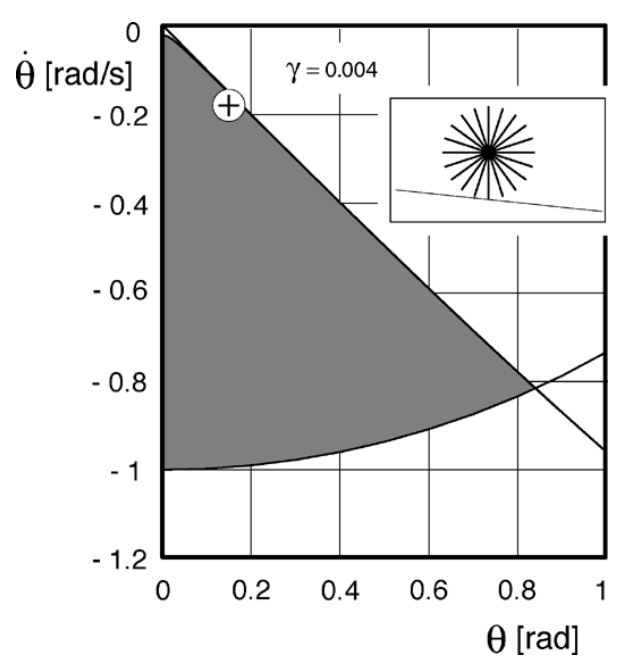

Fig. 6. Basin of attraction of the rimless wheel (model: see inset) with an interspoke angle of $0.3 \mathrm{rad}$. The initial conditions to the right of the dashed line can only be realized by starting at the top edge of a table. The "+" indicates the fixed point of this model at a slope of $\gamma=0.004 \mathrm{rad}$.

The fixed interspoke angle makes it impossible to start the rimless wheel with a larger initial leg angle than 0.15 rad, unless it were started at the top edge of a table. But even then, it would converge to its fixed point. Other than that, the only important gap in the basin of attraction is the small corner in the top, bounded by a line of constant energy through the point where Line (1) crosses $\theta=-0.15 \mathrm{rad}$. In that corner, the initial energy of the rimless wheel is enough to make it through the first step, but the fixed interspoke angle is too large to make it through the second.

In conclusion, only considering swing leg control, the maximally obtainable basin of attraction can be achieved with a controller mimicking the rimless wheel.

\section{Realistic Actuation Model}

The previous section indicates that the stability behavior of the rimless wheel is very close to the maximum achievable with swing leg control. So, now it is time to devise a simple but realistic form of actuation that mimics the rimless wheel behavior, acknowledging that instantaneous leg positioning is impossible. We propose to use a spring and a damper at the hip joint with a variable setpoint which can provide for an extra internal torque $T$ to the swing leg, extending the equations of motion to

$$
\begin{aligned}
& \text { with }\left[\begin{array}{l}
\ddot{\theta} \\
\ddot{\phi}
\end{array}\right]=\left[\begin{array}{l}
\sin (\theta-\gamma) \\
\sin (\phi)\left(\dot{\theta}^{2}-\cos (\theta-\gamma)\right)+\sin (\theta-\gamma)+T
\end{array}\right] \\
& T=-k\left(\phi-\phi_{\mathrm{sp}}\right)-c \dot{\phi} .
\end{aligned}
$$

The setpoint $\phi_{\mathrm{sp}}$ is set to $0.3 \mathrm{rad}$ corresponding to the fixed point of the passive walker at a slope of $\gamma=0.004 \mathrm{rad}$. The stiffness $k$ is the parameter that we vary, where $k=0$ corresponds to the fully passive simplest walker and $k=\infty$ corresponds to the rimless wheel. Note that $k$ is scaled with the foot mass $m$. The damping factor $c$ is set as a function of $k$ to provide critical damping

$$
c=2 \sqrt{k}
$$

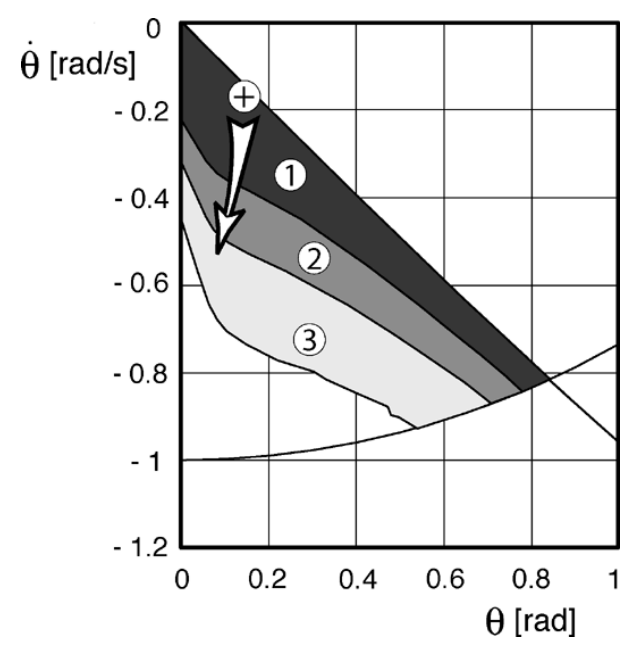

Fig. 7. Basin of attraction of the simplest walker with active hip spring. The setpoint of the hip spring is $\phi_{\mathrm{sp}}=0.3$ and critical damping is applied. The higher the hip spring stiffness, the larger the basin of attraction; $k=25$ leads to area (1), $k=50$ leads to area (2), and $k=100$ leads to area (3). The fixed point is for all three stiffness settings approximately the same, located at the "+." A disturbance from a step down in the floor would result in initial conditions away from the fixed point in the approximate direction of the white arrow.

Note that a physical realization of this type of actuation requires an active shift of the setpoint after each heel strike from $\phi_{\mathrm{sp}}$ to $-\phi_{\mathrm{sp}}$ or vice versa.

Fig. 7 presents the stability results for different stiffness values. A higher stiffness results in a faster swing leg motion and thus provides a better resistance against falling forward. The drawback is in energy consumption, but unfortunately the model with its massless feet is too much a simplification of real walking machines to allow for quantitative statements on energy expenditure. With this active hip spring stiffness we can arbitrarily make the basin of attraction as large as necessary up to complete coverage of the maximally obtainable area, so the problem of falling forward can be considered to be solved. Moreover, this is achieved without any feedback control other than a setpoint shift at heel strike.

Remember that this is just one possible way of speeding up the swing leg. It is not this particular implementation that counts, but the main idea behind it that the swing leg should be swung forward quickly, and then kept there at a not-too-large leg angle. We should emphasize here that, although simulated with a floor slope $\gamma=0.004 \mathrm{rad}$, this control rule works equally well for any slope larger than that. For smaller slopes, the preset leg angle should be decreased accordingly. Moreover, it appears that the amount of success of other dynamically walking bipeds can be attributed to how well their swing leg is brought forward, although the control rules are usually formulated implicitly (e.g., Westervelt et al. [25]).

\section{PROTOTYPE EXPERIMENTS}

\section{A. Mike}

We applied the proposed swing leg control to our prototype "Mike" (Fig. 1). Mike weighs $7 \mathrm{~kg}$ and measures $h \times b=0.7 \times$ $0.4 \mathrm{~m}$, and it uses $0.4 \mathrm{~g}$ of compressed $\mathrm{CO}_{2}$ per second. An elaborate description of Mike can be found in [28] while movie clips of Mike in action are available at our web site [16]. Mike 


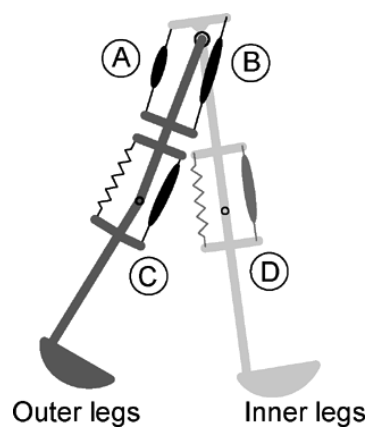

Fig. 8. Schematic structure and muscle attachments of Mike.

has four legs symmetrically paired, giving it approximate 2-D behavior. It differs from the simplest walking model by having knees, a distributed leg mass, round feet and by walking on a level floor (no slope!), all of which we will discuss in Section V.

Mike is actuated with a total of eight McKibben muscles; lightweight pneumatic actuators that act like springs with a stiffness proportional to the internal pressure [2], [22]. The McKibben muscles are arranged according to Fig. 8. The hip joint is actuated with two muscles (A) and two muscles (B) which are arranged as two antagonistic pairs, providing a combined joint stiffness. The knees are actively extended with McKibben muscles (C) and (D) which are counteracted by weak passive springs. There is no ankle actuation; the arc feet are rigidly attached to the shanks.

\section{B. Actuation System}

The McKibben muscles are fuelled from a 5.8- $\mathrm{MPa} \mathrm{CO}_{2}$ container via a two-stage pressure regulator and via electromagnetic valves that are activated by switches underneath the feet. The second-stage pressure regulator output is manually adjustable between 0.1 and $0.6 \mathrm{MPa}$ resulting in a hip joint stiffness up to $5 \mathrm{Nm} / \mathrm{rad}$ and a damping somewhat less than critical damping (estimated by observation). It is not feasible to perform a proper mapping between this stiffness in Mike and the scaled stiffness in the simplest walking model due to the extensive differences between the two, such as leg mass, foot arc radius, muscle nonlinearities and significant air flow dynamics. Therefore the comparison between the two will be of a qualitative nature only.

If a valve is switched "on," the muscle is filled from the pressure regulator output; if switched "off" it empties into atmosphere. For example, at activation of the inner leg foot switch, the outer knee muscles (muscle C in Fig. 8) are switched "off" to allow this knee to bend. A manually tuned $400 \mathrm{~ms}$ later they are switched back "on," ensuring a properly extended knee for the next step.

The proposed swing leg control in (10) is implemented by alternating the states of the antagonistic hip muscles. When the foot switch of the inner legs is activated, muscle B in Fig. 8 is switched "on" and muscle A is switched "off." At the next step this is inverted. As a result, the hip joint has a constant stiffness but a setpoint that alternates between $\phi_{\mathrm{sp}}$ and $-\phi_{\mathrm{sp}}$. The joint stiffness can be adjusted without altering the setpoint. We want to emphasize that there is no feedback control other than this once-per-step switching between preset muscle pressures. We dub this "feet-forward control."

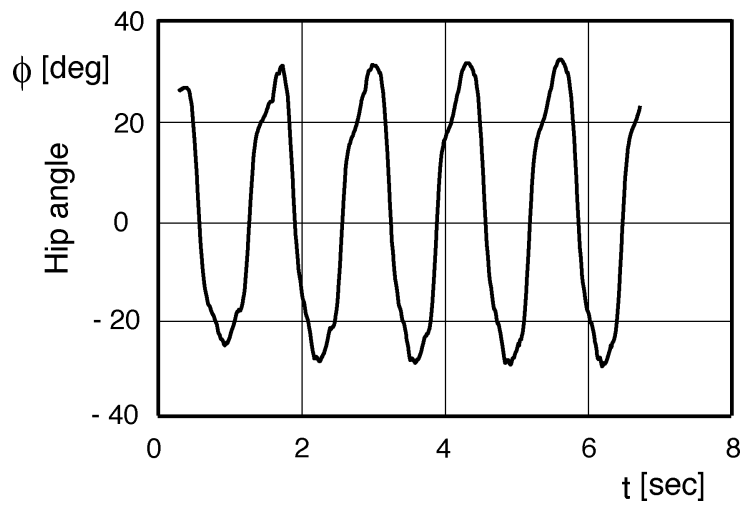

Fig. 9. Typical walking result with active hip muscles $(0.55 \mathrm{MPa})$ on a level floor. The prototype completes ten steps in this trial, showing convergence toward its fixed point after a manual launch.

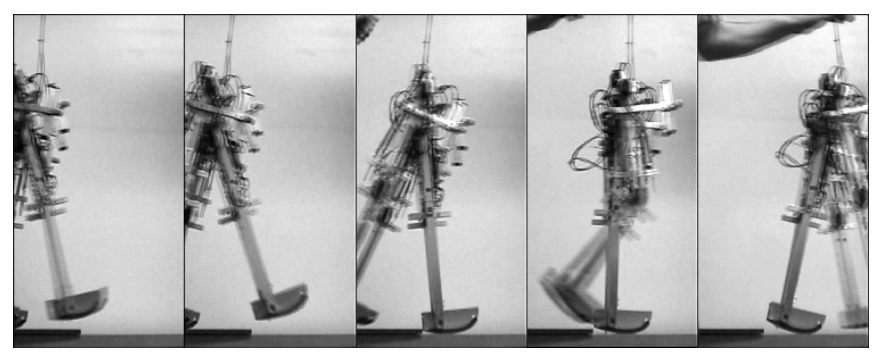

Fig. 10. Experiment with Mike walking on level floor and taking a step down as a representative disturbance.

\section{Stability Results}

Mike walks well. Fig. 9 shows a sample of the sustained gait for a hip muscle pressure of $0.55 \mathrm{MPa}$, see [16] for video evidence. We would have liked to create a figure of its basin of attraction like Fig. 7. However, the combined limitations on the number of experiments to be performed and on the physical possibilities to create controlled disturbances have led us to concentrate on one representative disturbance, namely a step-down. In the experiments the prototype walks steadily and then takes a step down of increasing height, see Fig. 10. Such a step down results in a larger stance leg velocity at the subsequent step as sketched with the white arrow in Fig. 7. The larger the step down height, the larger the arrow. If a larger hip muscle stiffness indeed allows a bigger step down, then our swing leg control rule is validated.

The stability results are shown in Fig. 11. A hip muscle pressure lower than $0.35 \mathrm{MPa}$ did not provide stable walking at all, not even without disturbances. When the pressure was increased, a larger step down could be handled. The muscles prohibit pressures higher than $0.55 \mathrm{MPa}$. Fig. 11 clearly shows a better robustness against falling forward with a higher hip pressure which corresponds to a higher joint stiffness.

\section{DISCUSSION}

\section{A. Level Floor}

One of the differences between Mike and the simplest walking model is that Mike's legs are not massless. When quickly moving the swing leg forward, it is not only rotated but its center of mass is lifted a little bit. For walkers with nonmassless legs, this provides a way of putting energy into the 


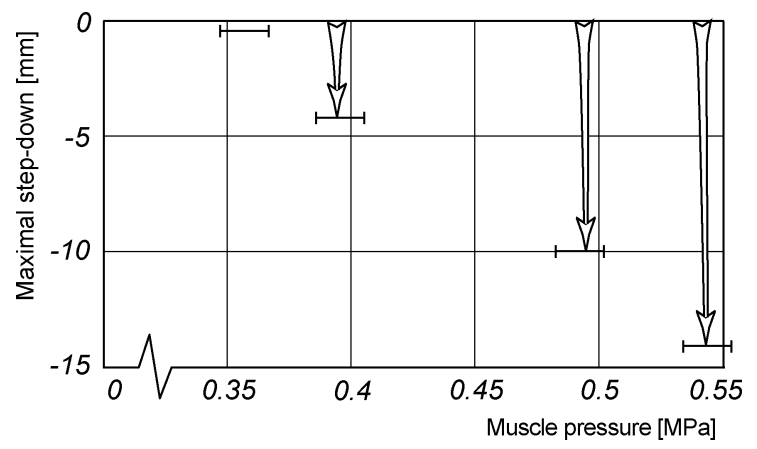

Fig. 11. Higher hip muscle pressure setting (corresponding to a higher hip joint stiffness) results in a larger step-down size and thus in a better resistance against disturbances. The arrows are depicted to indicate correspondence with the white arrow in Fig. 7.

system. For Mike this is sufficient to replace the gravitational energy that the simplest walking model obtained from walking downhill. As a result, Mike can walk on level terrain.

\section{B. Distributed Leg Mass}

Another difference between Mike and the simplest walking model is that the swing leg has a nonzero moment of inertia. For the simplest walker, any conceivable hip actuation would not influence the stance leg motion. For Mike, it does. The actual influence depends on the exact mass distribution of the swing leg. If its center of mass were located at the hip joint, the swing leg acceleration would have the adverse effect of a forward stance leg acceleration. With a center of mass more in the middle of the leg, the swing leg acceleration induces a backward acceleration (i.e., a deceleration) of the stance leg, which buys the prototype some extra time to place its swing leg. A linearized dynamic analysis of a straight-legged mechanism at midstance shows that such advantageous deceleration occurs if

$$
I<m c(l-c)
$$

with the leg moment of inertia $I$, leg mass $m$, leg length $l$, and the distance between the hip joint and the leg's center of mass c. From (12) we deduce that most normal constructions $(I \approx$ $\left.(1 / 12) m l^{2}\right)$ result in a slight (advantageous) deceleration of the hip.

Even though the stance leg and hip are decelerated, the center of mass of the entire system is accelerated forward due to the swing leg acceleration. This provides a neat opportunity to make the prototype start from a standstill, which was implemented successfully in the prototype.

\section{Feet}

The simplest walking model has point feet, whereas Mike's feet have a circular shape. Such feet provide extra robustness against the complementary problem of falling backward. As depicted in Figs. 4 and 5, the walker will fall backward if it has not enough velocity to overcome the vertical position. Circular feet smoothen the hip trajectory and thus relax the initial velocity requirement. As a result, the basin of attraction is enlarged in the upper right direction. We believe that round feet are a stability improvement measure complementary to the swing leg control rule proposed in this paper. However, a decisive study on the effect of circular feet on the basin of attraction has yet to be performed.

\section{Knees and Muscles}

Other differences between Mike and the simplest walking model are the knees and the nonlinear muscle properties. The knees do not essentially change the global behavior [18] as the swing leg starts and ends fully extended. One detail is that a violent knee extension at the end of the swing phase (e.g., as an automatic reaction to toe-stubbing) will decrease the hip angle. As at the end of the swing phase both the knee muscle and the corresponding hip muscle are activated, it seems advantageous to engage a biarticular muscle at that instant.

The muscles do not behave in an exactly linear fashion. Especially the damping and friction losses are strongly dependent on the muscle length, providing much more resistance when the muscle is close to its maximal extension. For Mike, this behavior is useful. The swing leg is brought forward without much resistance and is then effectively slowed down by the elongating hip muscle.

\section{E. Human Walking}

From measurements on human walking (e.g., Inman et al. [13], Winter [26]), it has been found that there is hip muscle activity that accelerates the swing leg at the beginning of the swing phase and decelerates it toward the end of the swing phase. Selles et al. [21] confirms that the human swing leg moves faster than it would if it were purely passive. At first sight, this seems like a waste of energy, but Kuo [15] hypothesizes that humans might speed up their swing leg to actually improve walking energetics, as it indirectly reduces the energy loss at heel strike. In addition to Kuo's hypothesis, the research in this paper provides a second hypothesis to why humans might speed up their swing leg; it increases the robustness against disturbances. It is not relevant what the exact muscle patterns are: as long as they result in an acceleration of the swing leg to a forward position, the positive robustness effect is similar to that of the control algorithm presented in this paper.

When comparing our proposed swing leg control rule to human muscle activation in more detail, it should be noted that our controller is always active, even if no disturbance is present. A more efficient controller would monitor (or even predict) the size of the disturbances and adjust the applied torques correspondingly. It seems reasonable to compare this to a walking human being who will violently throw forward his swing leg as a reflexive reaction to tripping. We hypothesize that this reflex is a 'full power' version of the stabilizing control rule presented in this paper, while in normal walking a 'low power' version is applied. This hypothesis is in line with Pratt [19] and Forner Cordero [7] who suggest that the maximum walking speed in humans is dictated by how fast one can swing his or her leg.

\section{CONCLUSION}

We started this research asking ourselves the question: "Can we achieve global stability for the simplest walking model with a simple swing leg control rule?" The answer is two-staged. 
First, we showed that swing leg control can only address the problem of falling forward. If the simplest walker falls backward, there is no way that any swing leg control can change this; there is simply not enough energy in the system to move past the vertical position.

Second, we showed that a simple controller can completely solve the problem of falling forward; all it needs to do is to get the swing leg timely in a forward position. A damped hip spring with a forward setpoint already suffices. The specific control and actuation details are not important as the same result can be achieved with any configuration if it is based on the following rule: "You will never fall forward if you put your swing leg fast enough in front of your stance leg. In order to prevent falling backward the next step, the swing leg shouldn't be too far in front." A controller designed according to this rule is easy to implement, because no a priori knowledge of the passive dynamic walking motion is needed.

We validated this rule with experiments with an autonomous, two-dimensional (four-legged) prototype with knees. The hip joint was actuated with McKibben muscles which provide a joint stiffness proportional to their internal $\mathrm{CO}_{2}$ pressure. By using only one muscle of a pair of antagonistic muscles, the hip joint was given a stiffness and a forward setpoint each step. In this manner, the swing leg was accelerated forward according to our proposed control rule. The prototype was made to take a step-down during a steady walk, and the maximal step-down height was recorded as a function of the hip muscle pressure (hip joint stiffness). It was shown that a higher pressure indeed allows a higher step-down. The resultant robust gait can be viewed at [16].

\section{ACKNOWLEDGMENT}

The authors would like to thank J.-D. Bakker and E. Mouw for providing a LART board for Mike's brain and J. van Frankenhuyzen for the mechanical work on Mike. The authors would like to thank J. Meijaard for his guidance toward the cell mapping method, and T. Platzer for an overview of the reflexes in human walking. Finally, they would also like to thank A. Ruina for many helpful comments and M. Garcia for supplying Figs. 2 and 3.

\section{REFERENCES}

[1] J. Adolfsson, H. Dankowicz, and A. Nordmark, "3d passive walkers: finding periodic gaits in the presence of discontinuities," Nonlinear Dyn., vol. 24, no. 2, pp. 205-229, 2001.

[2] C. P. Chou and B. Hannaford, "Measurement and modeling of mckibben pneumatic artificial muscles," IEEE Trans. Robot. Autom., vol. 12, no. 1, pp. 90-102, Feb. 1996.

[3] M. J. Coleman, "A Stability study of a three-dimensional passive-dynamic model of human gait," Ph.D. dissertation, Cornell Univ., Ithaca, NY, 1998.

[4] M. J. Coleman, A. Chatterjee, and A. Ruina, "Motions of a rimless spoked wheel: a simple 3d system with impacts," Dyn. Stability Syst., vol. 12, no. 3, pp. 139-160, 1997.

[5] M. J. Coleman and A. Ruina, "An uncontrolled toy that can walk but cannot stand still," Phys. Rev. Lett., vol. 80, no. 16, pp. 3658-3661, Apr. 1998.
[6] S. H. Collins, M. Wisse, and A. Ruina, "A two legged kneed passive dynamic walking robot," Proc. Int. J. Robot. Res., vol. 20, no. 7, pp. 607-615, Jul. 2001.

[7] A. F. Cordero, "Human Gait, Stumble and ... Fall?," Ph.D. dissertatiom, Univ. Twente, Enschede, The Netherlands, 2003.

[8] G. T. Fallis, "Walking toy ('improvement in walking toys')," U. S. Patent 376 588, Jan. 17, 1888.

[9] M. Garcia, A. Chatterjee, and A. Ruina, "Speed, efficiency, and stability of small-slope $2 \mathrm{~d}$ passive-dynamic bipedal walking," in Proc. IEEE Int. Conf. Robotics and Automation, Piscataway, NJ, 1998, pp. 2351-2356.

[10] M. Garcia, A. Chatterjee, A. Ruina, and M. J. Coleman, "The simplest walking model: stability, complexity, and scaling," ASME J. Biomech. Eng., vol. 120, no. 2, pp. 281-288, Apr. 1998.

[11] A. Goswami, B. Espiau, and A. Keramane, "Limit cycles and their stability in a passive bipedal gait," in Proc. IEEE Int. Conf. Robotics and Automation, Piscataway, NJ, 1996, pp. 246-251.

[12] C. S. Hsu, "Cell-to-cell mapping; a method of global analysis for nonlinear systems," in Applied Mathematical Sciences 64. New York: Springer-Verlag, 1987.

[13] V. T. Inman, H. J. Ralston, and F. Todd, Human Walking. Baltimore, MD: Williams \& Wilkinns, 1981.

[14] A. D. Kuo, "Stabilization of lateral motion in passive dynamic walking," Int. J. Robot. Res., vol. 18, no. 9, pp. 917-930, Sep. 1999.

[15] — , "Energetics of actively powered locomotion using the simplest walking model," J. Biomech. Eng., vol. 124, pp. 113-120, Feb. 2002.

[16] Delft Biorobotics Laboratory. (2003). [Online]. Available: http://dbl.tudelft.nl/

[17] T. McGeer, "Passive dynamic walking," Int. J. Robot. Res., vol. 9, no. 2, pp. 62-82, Apr. 1990.

[18] - "Passive walking with knees," in Proc. 1990 IEEE Int. Conf. Robotics and Automation, Los Alamitos, CA, 1990, pp. 1640-1645.

[19] J. E. Pratt, "Exploiting inherent robustness and natural dynamics in the control of bipedal walking robots," Ph.D. dissertation, MIT, Cambridge, MA, 2000.

[20] A. L. Schwab and M. Wisse, "Basin of attraction of the simplest walking model," presented at the Int. Conf. Noise and Vibration, 2001, Paper DETC2001/VIB-21 363.

[21] R. Selles, J. B. J. Bussmann, R. C. Wagenaar, and H. J. Stam, "Comparing predictive validity of four ballistic swing phase models of human walking," J. Biomech., vol. 34, pp. 1171-1177, 2001.

[22] R. Q. van der Linde, "Active leg compliance for passive walking," in Proc. IEEE Int. Conf. Robotics and Automation, May 1998, pp. 2339-2344.

[23] _ - "Passive bipedal walking with phasic muscle contraction," Biol. Cybern., vol. 81, no. 3, pp. 227-237, Sep. 1999.

[24] _ - "Bipedal walking with active springs, gait synthesis and prototype design," Ph.D. dissertation, Delft Univ. Technology, Delft, The Netherlands, Nov. 2001.

[25] E. R. Westervelt, J. W. Grizzle, and D. E. Koditschek, "Hybrid zero dynamics of planar biped walkers," IEEE Trans. Autom. Contr., vol. 48, no. 1, pp. 42-56, Jan. 2003.

[26] D. A. Winter, Biomechanics and Motor Control of Human Movement, 2nd ed. New York: Wileu, 1990.

[27] M. Wisse and A. L. Schwab, "A 3d passive dynamic biped with roll and yaw compensation," Robotica, vol. 19, pp. 275-284, 2001.

[28] M. Wisse and J. van Frankenhuyzen, "Design and construction of mike; a $2 \mathrm{~d}$ autonomous biped based on passive dynamic walking," presented at the Conf. Adaptive Motion of Animals and Machines, Kyoto, Japan, 2003, Paper WeP-I-1.

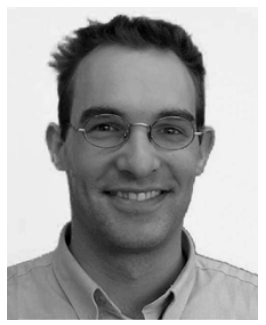

Martijn Wisse (M'02) received the M.S. and Ph.D degrees in mechanical engineering from Delft University of Technology, Delft, The Netherlands, in 2000 and 2004, respectively.

He held a postdoctoral position at the Robotics Institute, Carnegie Mellon University, Pittsburgh, PA, from 2004 to 2005. Since 2005, he has been an Assistant Professor at Delft University of Technology. His research interest is the field of humanoid robots, with a specialization in (passive) dynamic locomotion. 


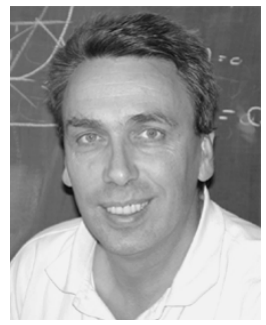

Arend L. Schwab received the M.S. and Ph.D. degrees in mechanical engineering from Delft University of Technology, Delft, The Netherlands, in 1983 and 2002, respectively.

$\mathrm{He}$ has been an Assistant Professor at Delft University of Technology since 1983. In 2002-2003, he worked as a Visiting Professor at the Department of Theoretical and Applied Mechanics, Cornell University, Ithaca, NY. His research interests include multibody dynamics, contact phenomena like nonholonomics and collisions, flexible multibody systems, finite element method, legged locomotion, and bicycle dynamics.

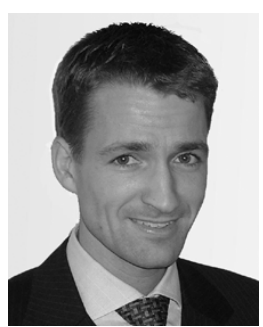

Richard Q. van der Linde received the M.Sc. and $\mathrm{Ph} . \mathrm{D}$. degrees (hons.) in mechanical engineering at the Delft University of Technology, Delft, The Netherlands, in 1995 and 2001, respectively.

He works part-time in industry and part-time as an Assistant Professor at the Delft University of Technology since 2002. His research interest is in the field of biorobotics, currently with a focus on two-legged walking robots and tele-operation. In industry, after holding a position as a Business Developer at FCS Control Systems, Hoofddorp, The Netherlands, he is currently employed as a Robotics Consultant for ALTRAN, Hoofddorp. The Netherlands.

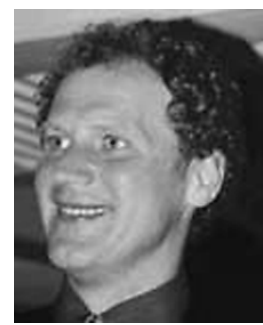

Frans C. T. van der Helm received the M.S. in human movement science from the Free University, Amsterdam, The Netherlands, in 1985, and the Ph.D. degree in mechanical engineering from Delft University of Technology, Delft, The Netherlands, in 1991.

He has worked at Delft University of Technology as an Assistant Professor (1990), Associate Professor (1993), and Full Professor (1999). He also holds a part-time full professorship at Twente University, Enschede, The Netherlands, since 1998. His research interest is in biomechanics of human motion, system identification and control theory, human motion control, muscle dynamics, musculoskeletal modeling, stability analysis for intrinsic and proprioceptive feedback, and finite element models for stress analysis of bone. 